\title{
Polarized Dirac fermions in de Sitter spacetime
}

\author{
Ion I. Cotăescu \\ West University of Timişoara, \\ V. Parvan Ave. 4, RO-1900 Timişoara
}

November 23, 2018

\begin{abstract}
The tetrad gauge invariant theory of the free Dirac field in two special moving charts of the de Sitter spacetime is investigated pointing out the operators that commute with the Dirac one. These are the generators of the symmetry transformations corresponding to isometries that give rise to conserved quantities according to the Noether theorem. With their help the plane wave spinor solutions of the Dirac equation with given momentum and helicity are derived and the final form of the quantum Dirac field is established. It is shown that the canonical quantization leads to a correct physical interpretation of the massive or massless fermion quantum fields.
\end{abstract}

Pacs: $04.62 .+\mathrm{v}$ 


\section{Introduction}

The recent astrophysical investigations showing that the expansion of the universe is accelerating [1] may increase the interest for the de Sitter spacetime which could represent the far future limit of the actual universe. On the other hand, the Dirac fermions (leptons and quarks) are the principal components of the matter since their gauge symmetries determine the main features of the physical picture. For these reasons, we believe that the study of the tetrad gauge invariant theory of the free Dirac field in de Sitter background may be important for understanding the influence of the external gravitational field minimally coupled with the fermion fields.

The Dirac equation in de Sitter spacetime (of radius $R=1 / \omega=\sqrt{3 / \Lambda_{c}}$, produced by the cosmological constant $\Lambda_{c}$ ) was studied in moving or static local charts (i.e., natural frames) suitable for separation of variables that should lead to significant analytical solutions [2, 3]. In [2] spherical wave solutions were derived in the local chart $\{t, r, \theta, \phi\}$ commonly associated to the Cartesian one $\{t, \vec{x}\}$ having the line element

$$
d s^{2}=d t^{2}-e^{2 \omega t} d \vec{x}^{2}
$$

in which the form of the plane wave solutions in a Cartesian tetrad gauge was outlined. Since in these moving charts the operator $i \partial_{t}$ is no longer a Killing vector field, the quantum modes corresponding to these solutions have no well-determined energies. Obviously, this is not an impediment but, in addition, there are some integration constants the physical meaning of which remains obscure. An alternative might be the particular solutions obtained through the separation of variables in static charts since these are energy eigenspinors. Recently, we found such solutions in a static central chart with the help of another version of Cartesian tetrad gauge [3]. These have integration constants with precise physical significance but, unfortunately, their form is too complicated to be normalized in the energy scale. Therefore, actually we do not have yet a complete set of particular solutions that may be used for writing the general form of the quantum field [4].

Looking for a such set of normalized particular solutions, we continue here the investigations of the free Dirac field in the chart with the line element (1). Our aim is to write down the plane wave solutions suggested in [2], defined as common eigenspinors of a complete set of commuting observables 
whose eigenvalues should determine the constants arising from the separation of variables. The main purpose of the present article is to show that these solutions are suitable for expressing the canonically quantized Dirac field in terms of creation and annihilation operators of fermions with good physical properties.

To this end we exploit the results of our theory of external symmetry [5] which explains the relations among the geometric symmetries and the operators commuting with the Dirac one written with the help of the Killing vectors long time ago [6]. In fact these operators are nothing other than the generators of the spinor representation of the universal covering group of the isometry group [5] and, therefore, they represent the main physical observables among which we can choose different sets of commuting operators defining quantum modes. This method is efficient especially in the case of the de Sitter spacetime where the high symmetry given by the $S O(4,1)$ isometry group [7, 8] offers one the opportunity of a very rich algebra of operators able to get physical meaning.

Our basic idea is that the significance of these observables is independent on the choice of the local chart and tetrad gauge, even though their form is strongly dependent on both these elements. For this reason we interpret the generators of the subgroup $E(3) \subset S O(4,1)$ as the three-dimensional momentum and angular momentum operators and take over the Hamiltonian operator found already in static frames [3, 5]. From this algebra we select the momentum operators and, in addition, we construct an one-component Pauli-Lubanski (or helicity) operator [9] obtaining thus the set of commuting observables that defines quantum modes with given momentum and helicity. We show that the common eigenspinors of these operators are the desired plane wave solutions of the Dirac equation which can be easily normalized in the momentum scale. Moreover, we demonstrate that the set of these solutions is complete (in generalized sense).

This set is used for expanding the free Dirac field in terms of creation and annihilation operators of fermions characterized by momentum and helicity, pointing out that the canonical quantization requires to adopt the standard anticommutation rules in momentum representation. In this way the conserved quantities predicted by the Noether theorem become the one-particle operators of the quantum field theory among them the diagonal ones are the momentum, helicity and charge operators. The Hamiltonian operator is not diagonal in this basis since it does not commute with the momentum 
components. The conclusion is that in our approach the second quantization can be done in canonical manner obtaining new results specific to the de Sitter geometry. However, the free fermions on this background have some properties similar to those found in Minkowski spacetime as, for example, the well-known law of neutrino polarization [泪.

We start in the second section with a brief review of the main results of our theory of external symmetry presenting the form of the symmetry generators and the conservation laws due to the Noether theorem. The moving chart we use here are introduced in section 3 where, in addition, we define a suitable basis of the $S O(4,1)$ generators helping us to identify the momentum, angular momentum and Hamiltonian operators. The plane wave solutions of the Dirac equation with fixed momentum and helicity are written in the next section while the section 5 is devoted to the canonical quantization of the Dirac field. After we present our concluding remarks, in an appendix we give the form of the isometries in the charts and parametrization used here and in another we give few useful formulas.

We use natural units with $\hbar=c=1$.

\section{Gauge and external symmetry}

In curved spacetimes, $M$, the choice of the local charts, $\{x\}$, of coordinates $x^{\mu}$ $(\mu, \nu, \ldots=0,1,2,3)$ is important from the observer point of view. Moreover, the tetrad gauge covariant theory of the fields with spin requires to explicitly use the tetrad fields, $e_{\hat{\mu}}(x)$ and $\hat{e}^{\hat{\mu}}(x)$, giving the local frames and the corresponding coframes. These are labeled by the local indices, $\hat{\mu}, \hat{\nu}, \ldots=0,1,2,3$, and have the orthonormalization properties $e_{\hat{\mu}} \cdot e_{\hat{\nu}}=\eta_{\hat{\mu} \hat{\nu}}, \hat{e}^{\hat{\mu}} \cdot \hat{e}^{\hat{\nu}}=\eta^{\hat{\mu} \hat{\nu}}$, and $\hat{e}^{\hat{\mu}} \cdot e_{\hat{\nu}}=\delta_{\hat{\nu}}^{\hat{\mu}}$, with respect to the Minkowski metric $\eta=\operatorname{diag}(1,-1,-1,-1)$. The 1-forms, $d \hat{x}^{\hat{\mu}}=\hat{e}_{\nu}^{\hat{\mu}} d x^{\nu}$, allow one to write the line element

$$
d s^{2}=\eta_{\hat{\mu} \hat{\nu}} d \hat{x}^{\hat{\mu}} d \hat{x}^{\hat{\nu}}=g_{\mu \nu}(x) d x^{\mu} d x^{\nu}
$$

defining the metric tensor $g_{\mu \nu}$ which raises or lowers the Greek indices while for the hated Greek ones we have to use the Minkowski metric. The derivatives in local frames are the vector fields $\hat{\partial}_{\hat{\nu}}=e_{\hat{\nu}}^{\mu} \partial_{\mu}$ which satisfy the com-

mutation rules $\left[\hat{\partial}_{\hat{\mu}}, \hat{\partial}_{\hat{\nu}}\right]=C_{\hat{\mu} \hat{\nu}} \because \hat{\sigma} \hat{\partial}_{\hat{\sigma}}$ giving the Cartan coefficients that help us to write the conecttion components in local frames. 
Let $\psi$ be a Dirac free field of mass $m$, defined on the space domain $D$, and $\bar{\psi}=\psi^{+} \gamma^{0}$ its Dirac adjoint. The tetrad gauge invariant action of the Dirac field minimally coupled with the gravitational field is

$$
\mathcal{S}[e, \psi]=\int d^{4} x \sqrt{g}\left\{\frac{i}{2}\left[\bar{\psi} \gamma^{\hat{\alpha}} D_{\hat{\alpha}} \psi-\left(\overline{D_{\hat{\alpha}} \psi}\right) \gamma^{\hat{\alpha}} \psi\right]-m \bar{\psi} \psi\right\}
$$

where $g=\left|\operatorname{det}\left(g_{\mu \nu}\right)\right|$ while $\gamma^{\hat{\alpha}}$ are the Dirac matrices which accomplish $\left\{\gamma^{\hat{\alpha}}, \gamma^{\hat{\beta}}\right\}=2 \eta^{\hat{\alpha} \hat{\beta}}$. The covariant derivatives in local frames, $D_{\hat{\alpha}}=\hat{\partial}_{\hat{\alpha}}+\hat{\Gamma}_{\hat{\alpha}}$, are expressed in terms of the spin connections

$$
\hat{\Gamma}_{\hat{\mu}}=\hat{\Gamma}_{\hat{\mu} \hat{\nu} \hat{\lambda}} S^{\hat{\nu} \hat{\lambda}}=\frac{i}{4}\left(C_{\hat{\mu} \hat{\nu} \hat{\lambda}}-C_{\hat{\mu} \hat{\lambda} \hat{\nu}}-C_{\hat{\nu} \hat{\lambda} \hat{\mu}}\right) S^{\hat{\nu} \hat{\lambda}}
$$

given by the basis-generators in covariant parametrization, $S^{\hat{\alpha} \hat{\beta}}=i\left[\gamma^{\hat{\alpha}}, \gamma^{\hat{\beta}}\right] / 4$, of the reducible spinor representation $\rho \sim(1 / 2,0) \oplus(0,1 / 2)$ of the $S L(2, \mathbb{C})$ group [9, 10] (i.e., the universal covering group of the Lorentz group, $L_{+}^{\uparrow}$, that is the gauge group of the metric $\eta$ ). The Dirac operator of the equation $E_{D} \psi=0$ derived from (3) reads $E_{D}=i \gamma^{\hat{\alpha}} D_{\hat{\alpha}}-m$. In other respects, from the conservation of the electric charge one deduces that when $e_{i}^{0}=0$ $(i, j, \ldots=1,2,3)$ then the time-independent relativistic scalar product of two spinors 11,

$$
\left\langle\psi, \psi^{\prime}\right\rangle=\int_{D} d^{3} x \mu(x) \bar{\psi}(x) \gamma^{0} \psi^{\prime}(x),
$$

has the weight function $\mu=\sqrt{g} e_{0}^{0}$.

The action (3) is gauge invariant in the sense that it remains unchanged when one performs a gauge transformation

$$
\begin{aligned}
\psi(x) & \rightarrow \psi^{\prime}(x)=\rho[A(x)] \psi(x) \\
e_{\hat{\alpha}}(x) & \rightarrow \quad e_{\hat{\alpha}}^{\prime}(x)=\Lambda_{\hat{\alpha} \cdot}^{\cdot \hat{\beta}}[A(x)] e_{\hat{\beta}}(x)
\end{aligned}
$$

produced by $A(x) \in S L(2, \mathbb{C})$ and $\Lambda[A(x)] \in L_{+}^{\uparrow}$. Based on this symmetry, we have defined the group of external symmetry, $S(M)$, corresponding to the isometry group $I(M)$. The transformations of $S(M)$ are isometries of $I(M)$, $x \rightarrow x^{\prime}=\phi_{\xi}(x)$ (depending on the parameters $\xi^{a}, a=1,2, \ldots$ ), combined with appropriate gauge transformations in such a manner to preserve the tetrad gauge. In a fixed gauge, one associates to each isometry $\phi_{\xi}$ the section $A_{\xi}(x) \in S L(2, \mathbb{C})$ defined by

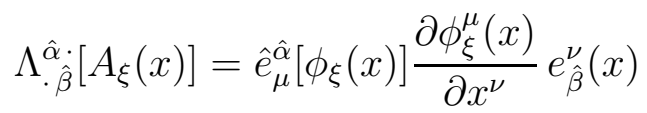


with the supplementary condition $A_{\xi=0}(x)=1 \in S L(2, C)$. Then the transformations of $S(M)$ are

$$
\begin{aligned}
& x \rightarrow x^{\prime}=\phi_{\xi}(x) \\
& e(x) \rightarrow e^{\prime}\left(x^{\prime}\right)=e\left[\phi_{\xi}(x)\right] \\
& \hat{e}(x) \rightarrow \hat{e}^{\prime}\left(x^{\prime}\right)=\hat{e}\left[\phi_{\xi}(x)\right] \\
& \psi(x) \rightarrow \psi^{\prime}\left(x^{\prime}\right)=\rho\left[A_{\xi}(x)\right] \psi(x) .
\end{aligned}
$$

In [5] we presented arguments that $S(M)$ is the universal covering group of $I(M)$ but the representation defined by the last of equations (9) is not an usual linear representation of $S(M)$ since it is induced by the representation $\rho$ of the group $S L(2, \mathbb{C})$ which, in general, differs from $S(M)$. For this reason we say that $\psi$ transforms according to the spinor representation of $S(M)$ induced by $\rho$.

The transformations (9) leave invariant the form of the operator $E_{D}$ in local frames. Consequently, each Killing vector, $k_{a}=\left(\partial_{\xi^{a}} \phi_{\xi}\right)_{\mid \xi=0}$, defines a basis-generator of the spinor representation [5],

$$
X_{a}=-i k_{a}^{\mu} D_{\mu}+\frac{1}{2} k_{a \mu ; \nu} e_{\hat{\alpha}}^{\mu} e_{\hat{\beta}}^{\nu} S^{\hat{\alpha} \hat{\beta}},
$$

which commutes with $E_{D}$. (Here the notation $; \nu$ stands for the usual covariant derivatives.) We must specify that this important result was obtained for the Dirac field in [6] without taking into account symmetry transformations. In [5] we have shown that the generators (10) satisfy the commutation relations

$$
\left[X_{a}, X_{b}\right]=i c_{a b c} X_{c}, \quad a, b, c=1,2, \ldots n
$$

given by the structure constants of $I(M)$. On the other hand, each generator can be split in orbital and spin parts as $X_{a}=L_{a}+S_{a}$ where the orbital terms,

$$
L_{a}=-i k_{a}^{\mu}(x) \partial_{\mu},
$$

are the basis-generators of the natural representation of $I(M)$ carried by the space of scalar functions over $M$. The spin terms

$$
S_{a}(x)=\frac{1}{2} \Omega_{a}^{\hat{\alpha} \hat{\beta}}(x) S_{\hat{\alpha} \hat{\beta}}
$$

are defined with the help of the functions

$$
\Omega_{a}^{\hat{\alpha} \hat{\beta}}=\left(\hat{e}_{\mu}^{\hat{\alpha}} \partial_{\nu} k_{a}^{\mu}+k_{a}^{\mu} \partial_{\mu} \hat{e}_{\nu}^{\hat{\alpha}}\right) e_{\hat{\lambda}}^{\nu} \eta^{\hat{\lambda} \hat{\beta}}
$$


that are antisymmetric if and only if $k_{a}$ is a Killing vector. Thus we see that the spin terms of the generators $X_{a}$ generally depend on $x$ and, therefore, they do not commute with the orbital terms. However, when $L_{a}$ and $S_{a}$ commute between themselves we say that the Dirac field transforms manifestly covariant under the transformations of the subgroup parametrized by $\xi^{a}$.

Our theory of external symmetry offers us the framework we need to calculate the conserved quantities predicted by the Noether theorem. Starting with the infinitesimal transformations of the one-parameter subgroup of $S(M)$ generated by $X_{a}$, we find that there exists the current $\Theta^{\mu}\left[X_{a}\right]$ which satisfies $\Theta^{\mu}\left[X_{a}\right]_{; \mu}=0$. For the action (3) this is

$$
\Theta^{\mu}\left[X_{a}\right]=-\tilde{T}_{\cdot \nu}^{\mu \cdot} k_{a}^{\nu}+\frac{1}{4} \bar{\psi}\left\{\gamma^{\hat{\alpha}}, S^{\hat{\beta} \hat{\gamma}}\right\} \psi e_{\hat{\alpha}}^{\mu} \Omega_{a \hat{\beta} \hat{\gamma}}
$$

where

$$
\tilde{T}_{\cdot \nu}^{\mu \cdot}=\frac{i}{2}\left[\bar{\psi} \gamma^{\hat{\alpha}} e_{\hat{\alpha}}^{\mu} \partial_{\nu} \psi-\left(\overline{\partial_{\nu} \psi}\right) \gamma^{\hat{\alpha}} e_{\hat{\alpha}}^{\mu} \psi\right]
$$

is a notation for a part of the stress-energy tensor of the Dirac field $\mathbb{7}$, [11. Finally, it is clear that the corresponding conserved quantity is the real number

$$
\int_{D} d^{3} x \sqrt{g} \Theta^{0}\left[X_{a}\right]=\frac{1}{2}\left[\left\langle\psi, X_{a} \psi\right\rangle+\left\langle X_{a} \psi, \psi\right\rangle\right] .
$$

We note that it is premature to interpret this formula as an expectation value or to speak about Hermitian conjugation of the operators $X_{a}$ with respect to (5) before to specify the boundary conditions on $D$. However, what is important here is that (17) is useful in quantization giving directly the one-particle operators of the quantum field theory.

\section{Observables in de Sitter spacetime}

Let us consider now $M$ be the de Sitter spacetime defined as the hyperboloid of radius $R$ in the five-dimensional flat spacetime $M^{5}$ of coordinates $Z^{A}$ $(A, B, \ldots=0,1,2,3,5)$ and metric $\eta^{5}=\operatorname{diag}(1,-1,-1,-1 .-1)$ [7]. The hyperboloid equation,

$$
\eta_{A B}^{5} Z^{A} Z^{B}=-R^{2},
$$

defines $M$ as an homogeneous space of the pseudo-orthogonal group $S O(4,1)$ which is in the same time the gauge group of the metric $\eta^{5}$ and the isometry 
group, $I(M)$, of the de Sitter spacetime. For this reason it is convenient to use the covariant real parameters $\xi^{A B}=-\xi^{B A}$ since then the orbital basisgenerators of the natural representation of $S O(4,1)$, carried by the space of scalar functions over $M^{5}$, have the standard form

$$
L_{A B}^{5}=i\left[\eta_{A C}^{5} Z^{C} \partial_{B}-\eta_{B C}^{5} Z^{C} \partial_{A}\right]
$$

They will give us directly the orbital basis-generators, $L_{(A B)}$, of the scalar representations of $I(M)$. Indeed, if one knows the functions $Z^{A}(x)$ solving (18) in the chart $\{x\}$ then one can write (19) in the form (12) finding the generators $L_{(A B)}$ and implicitly the components $k_{(A B)}^{\mu}(x)$ of the Killing vectors associated to the parameters $\xi^{A B}$ [5]. Furthermore, one has to calculate the spin parts $S_{(A B)}$, according to (13) and (14), obtaining the final form of the basis-generators $X_{(A B)}=L_{(A B)}+S_{(A B)}$ of the spinor representation of $S(M)$ induced by $\rho$.

In the de Sitter spacetime there are many static or moving charts of physical interest. Among the moving ones a special role plays the chart $\left\{t_{c}, \vec{x}\right\}$ with the conformal time $t_{c}$ and Cartesian spaces coordinates $x^{i}$ defined by

$$
\begin{aligned}
Z^{0} & =-\frac{1}{2 \omega^{2} t_{c}}\left[1-\omega^{2}\left(t_{c}{ }^{2}-r^{2}\right)\right] \\
Z^{5} & =-\frac{1}{2 \omega^{2} t_{c}}\left[1+\omega^{2}\left(t_{c}{ }^{2}-r^{2}\right)\right] \\
Z^{i} & =-\frac{1}{\omega t_{c}} x^{i}
\end{aligned}
$$

with $r=|\vec{x}|$. Even if this chart cover only a half of the manifold $M$, for $t_{c} \in(-\infty, 0)$ and $\vec{x} \in D \equiv \mathbb{R}^{3}$, it has the advantage of a simple conformal flat line element [11,

$$
d s^{2}=\frac{1}{\omega^{2} t_{c}^{2}}\left(d t_{c}^{2}-d \vec{x}^{2}\right)
$$

Moreover, the conformal time $t_{c}$ is related through

$$
\omega t_{c}=-e^{-\omega t}
$$

to the proper time $t \in(-\infty, \infty)$, of an observer at the point $\vec{x}=0$ of the chart $\{t, \vec{x}\}$ with the line element (1). In what follows we study the Dirac field 
in the chart $\{t, \vec{x}\}$ using the conformal time as a helpful auxiliary ingredient. The form of the line element (21) allows one to choose the simple Cartesian gauge with non-vanishing tetrad components [2]

$$
e_{0}^{0}=-\omega t_{c}, \quad e_{j}^{i}=-\delta_{j}^{i} \omega t_{c}, \quad \hat{e}_{0}^{0}=-\frac{1}{\omega t_{c}}, \quad \hat{e}_{j}^{i}=-\delta_{j}^{i} \frac{1}{\omega t_{c}}
$$

in which the Dirac operator reads

$$
\begin{aligned}
E_{D} & =-i \omega t_{c}\left(\gamma^{0} \partial_{t_{c}}+\gamma^{i} \partial_{i}\right)+\frac{3 i \omega}{2} \gamma^{0}-m \\
& =i \gamma^{0} \partial_{t}+i e^{-\omega t} \gamma^{i} \partial_{i}+\frac{3 i \omega}{2} \gamma^{0}-m
\end{aligned}
$$

and the weight function of the scalar product (5) is

$$
\mu=\left(-\omega t_{c}\right)^{-3}=e^{3 \omega t}
$$

The next step is to calculate the basis-generators $X_{(A B)}$ of the spinor representation of $S(M)$ in this gauge since, as mentioned, these commute with $E_{D}$. We observe that the group $S O(4,1)$ includes the subgroup $E(3)=$ $T(3)$ S $S O(3)$ of the isometries of the 3-dimensional Eucidean space (formed by $\mathbb{R}^{3}$ translations and proper rotations [9]) and, consequently, its universal covering group, $\tilde{E}(3)=T(3) \subseteq S U(2)$, is a subgroup of $S(M)$. The advantage of $\tilde{E}(3)$ is that its generators have the usual physical interpretation of momentum and total angular momentum operators. The problem of the Hamiltonian operator seems to be more complicated but we know that in the static central charts with the static time $t_{s}$ this is $H=\omega X_{(05)}=i \partial_{t_{s}}$ [ [5]. Thus the Hamiltonian operator and the components of momentum, $\vec{P}$, and total angular momentum, $\vec{J}\left(J^{i}=\varepsilon_{i j k} J_{j k} / 2\right)$, operators can be defined as

$$
\begin{aligned}
H & \equiv \omega X_{(05)}=-i \omega\left(t_{c} \partial_{t_{c}}+x^{i} \partial_{i}\right) \\
P^{i} & \equiv \omega\left(X_{(5 i)}-X_{(0 i)}\right)=-i \partial_{i} \\
J_{i j} & \equiv X_{(i j)}=-i\left(x^{i} \partial_{j}-x^{j} \partial_{i}\right)+S_{i j}
\end{aligned}
$$

remaining with the generators

$$
N^{i} \equiv X_{(5 i)}+X_{(0 i)}=\omega\left(t_{c}{ }^{2}-r^{2}\right) P^{i}+2 x^{i} H+2 \omega\left(S_{i 0} t_{c}+S_{i j} x^{j}\right)
$$


which do not have an obvious physical significance. The $S O(4,1)$ transformations corresponding to these generators and the associated isometries of the chart $\left\{t_{c}, \vec{x}\right\}$ are briefly presented in the Appendix A.

In the other local chart, $\{t, \vec{x}\}$, the Hamiltonian operator reads

$$
H=i \partial_{t}+\omega \vec{x} \cdot \vec{P}
$$

while the operators $\vec{P}$ and $\vec{J}=\vec{L}+\vec{S}$ (with $\vec{L}=\vec{x} \times \vec{P}$ ) do not change their form. Here the operators $K^{i} \equiv X_{(0 i)}$ are the analogous of the basis-generators of the Lorentz boosts of $S L(2, \mathbb{C})$ since in the limit of $\omega \rightarrow 0$, when (西) equals the Minkowski line element, the operators $H, P^{i}, J^{i}$ and $K^{i}$ become the generators of the spinor representation of the group $T(4) \subseteq S L(2, \mathbb{C})$ (i.e., the universal covering group of the Poincaré group [9, 10]).

In both these charts the generators (26)-(29) are self-adjoint with respect to the scalar product (5) with the weight function (25) if we consider usual boundary conditions on $D \equiv \mathbb{R}^{3}$. Therefore, for any generator $X$ we have

$$
\left\langle X \psi, \psi^{\prime}\right\rangle=\left\langle\psi, X \psi^{\prime}\right\rangle
$$

if (and only if) $\psi$ and $\psi^{\prime}$ are solutions of the Dirac equation which behave as tempered distributions or square integrable spinors with respect to (5). Moreover, all these operators commute with $E_{D}$ as well as any other operator from the algebra freely generated by them. We get thus a large collection of observables among which we can choose suitable sets of commuting operators defining the fermion quantum modes at the level of the relativistic quantum mechanics.

\section{Polarized plane waves solutions}

As suggested in [2], the plane wave solutions of the Dirac equation with $m \neq 0$ must be eigenspinors of the momentum operators $P^{i}$ corresponding to the eigenvalues $p^{i}$, with the same time modulation as the spherical waves. There-

fore, we have to look for particular solutions in the chart $\left\{t_{c}, \vec{x}\right\}$ involving either positive frequency plane waves or negative frequency ones. Bearing in mind that finally these must be related among themselves through the charge conjugation, we assume that, in the standard representation of the 
Dirac matrices (with diagonal $\gamma^{0}[10]$ ), these have the form

$$
\begin{aligned}
\psi_{\vec{p}}^{(+)} & =\left(\begin{array}{c}
f^{+}\left(t_{c}\right) \alpha(\vec{p}) \\
g^{+}\left(t_{c}\right) \frac{\vec{\sigma} \cdot \vec{p}}{p} \alpha(\vec{p})
\end{array}\right) e^{i \vec{p} \cdot \vec{x}} \\
\psi_{\vec{p}}^{(-)} & =\left(\begin{array}{c}
g^{-}\left(t_{c}\right) \frac{\vec{\sigma} \cdot \vec{p}}{p} \beta(\vec{p}) \\
f^{-}\left(t_{c}\right) \beta(\vec{p})
\end{array}\right) e^{-i \vec{p} \cdot \vec{x}}
\end{aligned}
$$

where $p=|\vec{p}|, \sigma_{i}$ denotes the Pauli matrices while $\alpha$ and $\beta$ are arbitrary Pauli spinors depending on $\vec{p}$. Replacing these spinors in the Dirac equation given by (24) and denoting $k=m / \omega$ and $\nu_{ \pm}=\frac{1}{2} \pm i k$, we find equations of the form (77) whose solutions can be written in terms of Hankel functions as

$$
\begin{aligned}
& f^{+}=\left(-f^{-}\right)^{*}=C t_{c}{ }^{2} e^{\pi k / 2} H_{\nu_{-}}^{(1)}\left(-p t_{c}\right) \\
& g^{+}=\left(-g^{-}\right)^{*}=C t_{c}{ }^{2} e^{-\pi k / 2} H_{\nu_{+}}^{(1)}\left(-p t_{c}\right) .
\end{aligned}
$$

The integration constant $C$ will be calculated from the ortonormalization condition in the momentum scale.

Hence the plane wave solutions are determined up to the significance of the Pauli spinors $\alpha$ and $\beta$. For $\vec{p} \neq 0$ these can be treated as in the flat case [4, 10] since, in the tetrad gauge (23), the spaces of these spinors carry unitary linear representations of the $\tilde{E}(3)$ group. Indeed, a transformation (9) produced by $\left(A, \phi_{A, \vec{a}}\right) \in \tilde{E}(3) \subset S(M)$ with $A \in S U(2)$ and $\vec{a} \in \mathbb{R}^{3}$, involves the linear isometry $\vec{x} \rightarrow \vec{x}^{\prime}=\Lambda(A) \vec{x}+\vec{a}$ and the global transformation $\psi(t, \vec{x}) \rightarrow \psi^{\prime}\left(t, \vec{x}^{\prime}\right)=\rho(A) \psi(t, \vec{x})$. Consequently, the Pauli spinors transform according to the unitary (linear) representation

$$
\alpha(\vec{p}) \rightarrow e^{-i \vec{a} \cdot \vec{p}} A \alpha\left[\Lambda(A)^{-1} \vec{p}\right]
$$

(and similarly for $\beta$ ) that conserves the orthogonality. This means that any pair of orthogonal spinors, $\tilde{\xi}_{\sigma}(\vec{p}), \sigma= \pm 1 / 2$, (obeying $\tilde{\xi}_{\sigma}^{+} \tilde{\xi}_{\sigma^{\prime}}=\delta_{\sigma, \sigma^{\prime}}$ ), represents an arbitrary basis in the space of Pauli spinors

$$
\alpha(\vec{p})=\sum_{\sigma} \tilde{\xi}_{\sigma}(\vec{p}) a(\vec{p}, \sigma)
$$

whose components, $a(\vec{p}, \sigma)$, are the wave functions in momentum representation of a particle of momentum $\vec{p}$ and polarization $\sigma$. According to the 
standard interpretation of the negative frequency terms [4, 10], the corresponding basis of the space of $\beta$ spinors is defined by

$$
\beta(\vec{p})=\sum_{\sigma} \tilde{\eta}_{\sigma}(\vec{p})[b(\vec{p}, \sigma)]^{*}, \quad \tilde{\eta}_{\sigma}(\vec{p})=i \sigma_{2}\left[\tilde{\xi}_{\sigma}(\vec{p})\right]^{*}
$$

where $b(\vec{p}, \sigma)$ are the antiparticle wave functions. It remains to choose the concrete basis we need, using supplementary physical assumptions. Since it is not sure that the so called spin basis [⿴囗十 can be correctly defined in de Sitter geometry, we prefer the helicity basis. This is formed by the orthogonal Pauli spinors of helicity $\lambda= \pm 1 / 2$ which fulfill

$$
\vec{\sigma} \cdot \vec{p} \tilde{\xi}_{\lambda}(\vec{p})=2 p \lambda \tilde{\xi}_{\lambda}(\vec{p}), \quad \vec{\sigma} \cdot \vec{p} \tilde{\eta}_{\lambda}(\vec{p})=-2 p \lambda \tilde{\eta}_{\lambda}(\vec{p}) .
$$

The desired particular solutions of the Dirac equation with $m \neq 0$ result from (32) and (33) where we replace (34) and (35) and the spinors (37) and (38) written in the helicity basis (39). It remains to calculate the normalization constant $C$ with respect to the scalar product (5) with the weight function (25). After few manipulations in the chart $\{t, \vec{x}\}$, it turns out the final form of the fundamental spinor solutions of positive and negative frequencies with momentum $\vec{p}$ and helicity $\lambda$,

$$
\begin{aligned}
& U_{\vec{p}, \lambda}(t, \vec{x})=i N\left(\begin{array}{c}
\frac{1}{2} e^{\pi k / 2} H_{\nu_{-}}^{(1)}\left(q e^{-\omega t}\right) \tilde{\xi}_{\lambda}(\vec{p}) \\
\lambda e^{-\pi k / 2} H_{\nu_{+}}^{(1)}\left(q e^{-\omega t}\right) \tilde{\xi}_{\lambda}(\vec{p})
\end{array}\right) e^{i \vec{p} \cdot \vec{x}-2 \omega t} \\
& V_{\vec{p}, \lambda}(t, \vec{x})=i N\left(\begin{array}{c}
-\lambda e^{-\pi k / 2} H_{\nu_{-}}^{(2)}\left(q e^{-\omega t}\right) \tilde{\eta}_{\lambda}(\vec{p}) \\
\frac{1}{2} e^{\pi k / 2} H_{\nu_{+}}^{(2)}\left(q e^{-\omega t}\right) \tilde{\eta}_{\lambda}(\vec{p})
\end{array}\right) e^{-i \vec{p} \cdot \vec{x}-2 \omega t},
\end{aligned}
$$

which depend on the new parameter $q=p / \omega$ and

$$
N=\frac{1}{(2 \pi)^{3 / 2}} \sqrt{\pi q} .
$$

Using (76) and (78), it is not hard to verify that these spinors are chargeconjugated to each other,

$$
V_{\vec{p}, \lambda}=\left(U_{\vec{p}, \lambda}\right)^{c}=C\left(\bar{U}_{\vec{p}, \lambda}\right)^{T}, \quad C=i \gamma^{2} \gamma^{0}
$$

satisfy the ortonormalization relations

$$
\begin{aligned}
& \left\langle U_{\vec{p}, \lambda}, U_{\vec{p}^{\prime}, \lambda^{\prime}}\right\rangle=\left\langle V_{\vec{p}, \lambda}, V_{\vec{p}^{\prime}, \lambda^{\prime}}\right\rangle=\delta_{\lambda \lambda^{\prime}} \delta^{3}\left(\vec{p}-\vec{p}^{\prime}\right), \\
& \left\langle U_{\vec{p}, \lambda}, V_{\vec{p}^{\prime}, \lambda^{\prime}}\right\rangle=\left\langle V_{\vec{p}, \lambda}, U_{\vec{p}^{\prime}, \lambda^{\prime}}\right\rangle=0
\end{aligned}
$$


and represent a complete set of solutions in the sense that

$$
\int d^{3} p \sum_{\lambda}\left[U_{\vec{p}, \lambda}(t, \vec{x}) U_{\vec{p}, \lambda}^{+}\left(t, \vec{x}^{\prime}\right)+V_{\vec{p}, \lambda}(t, \vec{x}) V_{\vec{p}, \lambda}^{+}\left(t, \vec{x}^{\prime}\right)\right]=e^{-3 \omega t} \delta^{3}\left(\vec{x}-\vec{x}^{\prime}\right) .
$$

Let us observe that the factor $e^{-3 \omega t}$ does not have a special physical meaning since its role is only to compensate the weight function (25). Other important properties are

$$
\begin{array}{rlrl}
P^{i} U_{\vec{p}, \lambda} & =p^{i} U_{\vec{p}, \lambda}, & & P^{i} V_{\vec{p}, \lambda}=-p^{i} V_{\vec{p}, \lambda}, \\
W U_{\vec{p}, \lambda} & =p \lambda U_{\vec{p}, \lambda}, & W V_{\vec{p}, \lambda}=-p \lambda V_{\vec{p}, \lambda} .
\end{array}
$$

where $W=\vec{J} \cdot \vec{P}=\vec{S} \cdot \vec{P}$ is the helicity operator which is analogous to the time-like component of the four-component Pauli-Lubanski operator of the Poincaré algebra [9]. Thus, we arrive at the conclusion that the fundamental solutions (40) and (41) form a complete set of common eigenspinors of the operators $P^{i}$ and $W$.

In the case of $m=0$ (when $k=0$ ) it is convenient to consider the chiral representation of the Dirac matrices (with diagonal $\gamma^{5}[\llbracket$ ) and the

chart $\left\{t_{c}, \vec{x}\right\}$. We find that the fundamental solutions in helicity basis of the left-handed massless Dirac field,

$$
\begin{aligned}
U_{\vec{p}, \lambda}^{0}\left(t_{c}, \vec{x}\right) & =\lim _{k \rightarrow 0} \frac{1-\gamma^{5}}{2} U_{\vec{p}, \lambda}\left(t_{c}, \vec{x}\right) \\
& =\left(\frac{-\omega t_{c}}{2 \pi}\right)^{3 / 2}\left(\begin{array}{c}
\left(\frac{1}{2}-\lambda\right) \tilde{\xi}_{\lambda}(\vec{p}) \\
0
\end{array}\right) e^{-i p t_{c}+i \vec{p} \cdot \vec{x}} \\
V_{\vec{p}, \lambda}^{0}\left(t_{c}, \vec{x}\right) & =\lim _{k \rightarrow 0} \frac{1-\gamma^{5}}{2} V_{\vec{p}, \lambda}\left(t_{c}, \vec{x}\right) \\
& =\left(\frac{-\omega t_{c}}{2 \pi}\right)^{3 / 2}\left(\begin{array}{c}
\left(\frac{1}{2}+\lambda\right) \tilde{\eta}_{\lambda}(\vec{p}) \\
0
\end{array}\right) e^{i p t_{c}-i \vec{p} \cdot \vec{x}}
\end{aligned}
$$

are non-vanishing only for positive frequency and $\lambda=-1 / 2$ or negative frequency and $\lambda=1 / 2$, as in Minkowski spacetime. Obviously, these solutions have similar properties as (43)-(48).

\section{Quantization}

The quantization can be done simply considering that the wave functions in momentum representation, $a(\vec{p}, \lambda)$ and $b(\vec{p}, \lambda)$, become field operators (so 
that $\left.b^{*} \rightarrow b^{\dagger}\right)$. Then the quantum field which satisfies the Dirac equation with $m \neq 0$ in the chart $\{t, \vec{x}\}$ reads

$$
\begin{aligned}
\psi(t, \vec{x}) & =\psi^{(+)}(t, \vec{x})+\psi^{(-)}(t, \vec{x}) \\
& =\int d^{3} p \sum_{\lambda}\left[U_{\vec{p}, \lambda}(x) a(\vec{p}, \lambda)+V_{\vec{p}, \lambda}(x) b^{\dagger}(\vec{p}, \lambda)\right] .
\end{aligned}
$$

We believe that the particle $\left(a, a^{\dagger}\right)$ and antiparticle $\left(b, b^{\dagger}\right)$ operators must fulfill the standard anticommutation relations in the momentum representation, from which the non-vanishing ones are

$$
\left\{a(\vec{p}, \lambda), a^{\dagger}\left(\vec{p}^{\prime}, \lambda^{\prime}\right)\right\}=\left\{b(\vec{p}, \lambda), b^{\dagger}\left(\vec{p}^{\prime}, \lambda^{\prime}\right)\right\}=\delta_{\lambda, \lambda^{\prime}} \delta^{3}\left(\vec{p}-\vec{p}^{\prime}\right)
$$

since then the equal-time anticommutator takes the canonical form

$$
\left\{\psi(t, \vec{x}), \bar{\psi}\left(t, \vec{x}^{\prime}\right)\right\}=e^{-3 \omega t} \gamma^{0} \delta^{3}\left(\vec{x}-\vec{x}^{\prime}\right),
$$

as it results from (46). In general, the partial anticommutator functions,

$$
\tilde{S}^{( \pm)}\left(t, t^{\prime}, \vec{x}-\vec{x}^{\prime}\right)=i\left\{\psi^{( \pm)}(t, \vec{x}), \bar{\psi}^{( \pm)}\left(t^{\prime}, \vec{x}^{\prime}\right)\right\},
$$

and the total one $\tilde{S}=\tilde{S}^{(+)}+\tilde{S}^{(-)}$are rather complicated since for $t \neq t^{\prime}$ we have no more identities like (78) which should simplify their time-dependent parts. In any event, these are solutions of the Dirac equation in both their sets of coordinates and help one to write the Green functions in usual manner. For example, from the standard definition of the Feynman propagator [4],

$$
\begin{aligned}
& \tilde{S}_{F}\left(t, t^{\prime}, \vec{x}-\vec{x}^{\prime}\right)=i\left\langle 0\left|T\left[\psi(x) \bar{\psi}\left(x^{\prime}\right)\right]\right| 0\right\rangle \\
& =\theta\left(t-t^{\prime}\right) \tilde{S}^{(+)}\left(t, t^{\prime}, \vec{x}-\vec{x}^{\prime}\right)-\theta\left(t^{\prime}-t\right) \tilde{S}^{(-)}\left(t, t^{\prime}, \vec{x}-\vec{x}^{\prime}\right),
\end{aligned}
$$

we find that

$$
E_{D}(x) \tilde{S}_{F}\left(t, t^{\prime}, \vec{x}-\vec{x}^{\prime}\right)=-e^{-3 \omega t} \delta^{4}\left(x-x^{\prime}\right) .
$$

Another argument for this quantization procedure is that the one-particle operators given by the Noether theorem have similar structures and properties like those of the quantum theory of the free fields in flat spacetime. Indeed, according to (17) and (31), the Noether theorem guarantees that for any generator $X$ of the spinor representation of $S(M)$ there exists a 
conserved one-particle operator of the quantum field theory which can be calculated simply as

$$
\mathbf{X}=:\langle\psi, X \psi\rangle \text { : }
$$

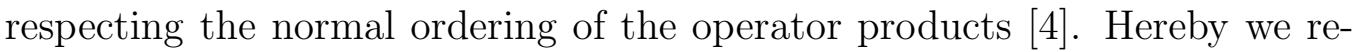
cover the standard algebraic properties

$$
[\mathbf{X}, \psi(x)]=-X \psi(x), \quad\left[\mathbf{X}, \mathbf{X}^{\prime}\right]=:\left\langle\psi,\left[X, X^{\prime}\right] \psi\right\rangle:
$$

due to the canonical quantization adopted here.

The diagonal one-particle operators result directly from (58) and (44)(48). In this way we obtain the momentum components

$$
\mathbf{P}^{i}=:\left\langle\psi, P^{i} \psi\right\rangle:=\int d^{3} p p^{i} \sum_{\lambda}\left[a^{\dagger}(\vec{p}, \lambda) a(\vec{p}, \lambda)+b^{\dagger}(\vec{p}, \lambda) b(\vec{p}, \lambda)\right]
$$

and the Pauli-Lubanski operator

$$
\mathbf{W}=:\langle\psi, W \psi\rangle:=\int d^{3} p \sum_{\lambda} p \lambda\left[a^{\dagger}(\vec{p}, \lambda) a(\vec{p}, \lambda)+b^{\dagger}(\vec{p}, \lambda) b(\vec{p}, \lambda)\right] .
$$

The definition (58) holds for the generators of internal symmetries too, including the particular case of $X=1$ when the bracket

$$
\mathbf{Q}=:\langle\psi, \psi\rangle:=\int d^{3} p \sum_{\lambda}\left[a^{\dagger}(\vec{p}, \lambda) a(\vec{p}, \lambda)-b^{\dagger}(\vec{p}, \lambda) b(\vec{p}, \lambda)\right]
$$

gives just the charge operator corresponding to the internal $U(1)$ symmetry of (3) [10, 11]. It is obvious that all these operators are generators of the external or internal symmetry transformations of the quantum fields [4]. The conclusion is that the helicity states of the Fock space are common eigenstates of the set $\left\{\mathbf{Q}, \mathbf{P}^{i}, \mathbf{W}\right\}$.

The Hamiltonian operator $\mathbf{H}=:\langle\psi, H \psi\rangle$ : is conserved but is not diagonal in this basis since it does not commute with $\mathbf{P}^{i}$ and $\mathbf{W}$. Its form in momentum representation can be calculated using the identity

$$
H U_{\vec{p}, \lambda}(t, \vec{x})=-i \omega\left(p^{i} \partial_{p^{i}}+\frac{3}{2}\right) U_{\vec{p}, \lambda}(t, \vec{x})
$$

and the similar one for $V_{\vec{p}, \lambda}$, leading to

$$
\mathbf{H}=\frac{i \omega}{2} \int d^{3} p p^{i} \sum_{\lambda}\left[a^{\dagger}(\vec{p}, \lambda) \stackrel{\leftrightarrow}{\partial}_{p^{i}} a(\vec{p}, \lambda)+b^{\dagger}(\vec{p}, \lambda) \stackrel{\leftrightarrow}{\partial}_{p^{i}} b(\vec{p}, \lambda)\right]
$$


where the derivatives act as $f \stackrel{\leftrightarrow}{\partial} h=f \partial h-(\partial f) h$. Hereby it results the expected behavior of $\mathbf{H}$ under the space translations of $\tilde{E}(3)$ which transform the operators $a$ and $b$ according to (36). Moreover, it is worth pointing out that, there exist momentum dependent phase transformations

$$
\begin{aligned}
U_{\vec{p}, \lambda} & \rightarrow e^{i \chi(\vec{p})} U_{\vec{p}, \lambda} \\
V_{\vec{p}, \lambda} & \rightarrow e^{-i \chi(\vec{p})} V_{\vec{p}, \lambda} \\
a(\vec{p}, \lambda) & \rightarrow e^{-i \chi(\vec{p})} a(\vec{p}, \lambda) \\
b(\vec{p}, \lambda) & \rightarrow e^{-i \chi(\vec{p})} b(\vec{p}, \lambda)
\end{aligned}
$$

(with $\chi \in \mathbb{R}$ ) leaving invariant the operators $\psi, \mathbf{Q}, \mathbf{P}^{i}$ and $\mathbf{W}$ as well as the equations (52), while the Hamiltonian operator transforms as

$$
\mathbf{H} \rightarrow \mathbf{H}+\omega \int d^{3} p\left[p^{i} \partial_{p^{i}} \chi(\vec{p})\right] \sum_{\lambda}\left[a^{\dagger}(\vec{p}, \lambda) a(\vec{p}, \lambda)+b^{\dagger}(\vec{p}, \lambda) b(\vec{p}, \lambda)\right] .
$$

This interesting property may be interpreted as a new type of gauge transformations depending on momentum instead of coordinates. Our preliminary calculations indicate that this gauge may be helpful for analyzing the behavior of our theory near $\omega \sim 0$.

In the simpler case of the left-handed massless field which has the fundamental spinor solutions (49) and (50) we obtain similar results among them we recover the standard rule of neutrino polarization.

\section{Concluding remarks}

We have derived here a complete set of normalized plane wave solutions of the Dirac equation in the chart with the line element (1) of the de Sitter spacetime. These determine the quantum modes of polarized free fermions characterized by momentum and helicity. Thus we obtain a similar approach as in special relativity allowing us to perform the second quantization of the free Dirac field according to the canonical method.

It remains to solve many associated problems from the pure mathematical ones up to those regarding to the physical interpretation of the specific observables of the de Sitter background. First of all, one has to look for an orbital analysis analogous to the Wigner theory of the induced representations of the Poincaré group [9, 10]. This could help one to understand the meaning 
of the rest frames (with $\vec{p}=0$ ) in the de Sitter geometry indicating which are the "booster" mechanisms giving rise to waves of arbitrary momentum from those with $\vec{p}=0$. In the same time it is important to investigate the physical consequences of the relations among the Hamiltonian operator and the other generators of the spinor representation of the $S(M)$ group and the role and significance of the gauge transformations (65)-(69). Moreover, in further investigations of the quantum free Dirac field, some specific problems could appear but these seem to be rather technical, e.g. the properties of commutator and Green functions, calculation of the action of more complicated conserved operators, evaluation of the inertial effects etc. However, in our opinion, the next important step would be to construct a similar theory for the free electromagnetic field which should complete the basic ingredients one needs for developing the perturbative QED in de Sitter spacetime.

The results obtained here show that, even though many particular fea-

tures of the quantum theory in curved spacetimes depend on the choice of the local chart and tetrad gauge, there are covariance properties providing us with operators with invariant commutation relations. For this reason we hope that our approach based on external symmetries could be an argument for a general tetrad gauge covariant theory of quantum fields with spin in which the second quantization should be independent on the frames where one works.

\section{A $\mathrm{SO}(4,1)$ transformations and isometries}

The spacetime $M^{5}$ is pseudo-Eucidean with the metric $\eta^{5}$ that is invariant under the coordinate transformations $Z^{A} \rightarrow{ }^{5} \Lambda_{\cdot B}^{A} Z^{B}$ where ${ }^{5} \Lambda \in S O(4,1)$. Each coordinate transformation give rise to an isometry of $M$ which can be calculated in the local chart $\left\{t_{c}, \vec{x}\right\}$ using the equations $(20)$. We remind the reader that the basis generators ${ }^{5} X_{A B}$ of the fundamental (linear) representation of $S O(4,1)$, carried by $M^{5}$, have the matrix elements

$$
\left({ }^{5} X_{A B}\right)_{\cdot D}^{C \cdot}=i\left(\delta_{A}^{C} \eta_{B D}-\delta_{B}^{C} \eta_{A D}\right)
$$

The transformations of $S O(3) \subset S O(4,1)$ are simple rotations of $Z^{i}$ and $x^{i}$ which transform alike since this symmetry is global. For the other transformations generated by $H, P^{i}$ and $N^{i}$ the linear transformations in $M^{5}$ and 
the isometries are different. Those due to $H$,

$$
\begin{aligned}
Z^{0} & \rightarrow Z^{0} \cosh \alpha-Z^{5} \sinh \alpha \\
e^{-i \xi_{H}{ }^{5} H}: \quad Z^{5} & \rightarrow-Z^{5} \sinh \alpha+Z^{0} \cosh \alpha \\
& Z^{i} \rightarrow Z^{i}
\end{aligned}
$$

where $\alpha=\omega \xi_{H}$, produce the dilatations $t_{c} \rightarrow t_{c} e^{\alpha}$ and $x^{i} \rightarrow x^{i} e^{\alpha}$, while the transformations

$$
\begin{aligned}
Z^{0} & \rightarrow Z^{0}+\omega \vec{\xi}_{P} \cdot \vec{Z}+\frac{1}{2} \omega^{2} \xi_{P}{ }^{2}\left(Z^{0}+Z^{5}\right) \\
e^{-i \vec{\xi}_{P} \cdot{ }^{5} \vec{P}}: \quad Z^{5} & \rightarrow Z^{5}-\omega \vec{\xi}_{P} \cdot \vec{Z}-\frac{1}{2} \omega^{2} \xi_{P}^{2}\left(Z^{0}+Z^{5}\right) \\
Z^{i} & \rightarrow Z^{i}+\omega \xi_{P}^{i}\left(Z^{0}+Z^{5}\right)
\end{aligned}
$$

give the space translations $x^{i} \rightarrow x^{i}+\xi_{P}^{i}$ at fixed $t_{c}$. More interesting are the transformations

$$
\begin{aligned}
Z^{0} & \rightarrow Z^{0}-\vec{\xi}_{N} \cdot \vec{Z}+\frac{1}{2} \xi_{N}^{2}\left(Z^{0}-Z^{5}\right) \\
e^{-i \vec{\xi}_{N} \cdot \vec{N}_{N}}: \quad Z^{5} & \rightarrow Z^{5}-\vec{\xi}_{N} \cdot \vec{Z}+\frac{1}{2} \xi_{N}^{2}\left(Z^{0}-Z^{5}\right) \\
& Z^{i} \rightarrow Z^{i}-\xi_{N}^{i}\left(Z^{0}-Z^{5}\right)
\end{aligned}
$$

which lead to the isometries

$$
\begin{aligned}
t_{c} & \rightarrow \frac{t_{c}}{1-2 \omega \vec{\xi}_{N} \cdot \vec{x}-\omega^{2} \xi_{N}^{2}\left(t_{c}^{2}-r^{2}\right)} \\
x^{i} & \rightarrow \frac{x^{i}+\omega \xi_{N}^{i}\left(t_{c}^{2}-r^{2}\right)}{1-2 \omega \vec{\xi}_{N} \cdot \vec{x}-\omega^{2} \xi_{N}{ }^{2}\left(t_{c}{ }^{2}-r^{2}\right)} .
\end{aligned}
$$

We denoted here $\xi_{P}{ }^{2}=\left(\vec{\xi}_{P}\right)^{2}$ and $\xi_{N}{ }^{2}=\left(\vec{\xi}_{N}\right)^{2}$.

\section{B Some properties of Hankel functions}

According to the general properties of the Hankel functions [12, we deduce that those used here, $H_{\nu_{ \pm}}^{(1,2)}(z)$, with $\nu_{ \pm}=\frac{1}{2} \pm i k$ and $z \in \mathbb{R}$, are related among themselves through

$$
\left[H_{\nu_{ \pm}}^{(1,2)}(z)\right]^{*}=H_{\nu_{\mp}}^{(2,1)}(z)
$$

satisfy the equations

$$
\left(\frac{d}{d z}+\frac{\nu_{ \pm}}{z}\right) H_{\nu_{ \pm}}^{(1,2)}(z)=i e^{ \pm \pi k} H_{\nu_{\mp}}^{(1,2)}(z)
$$


and the identities

$$
e^{ \pm \pi k} H_{\nu_{\mp}}^{(1)}(z) H_{\nu_{ \pm}}^{(2)}(z)+e^{\mp \pi k} H_{\nu_{ \pm}}^{(1)}(z) H_{\nu_{\mp}}^{(2)}(z)=\frac{4}{\pi z}
$$

\section{References}

[1] N. Trentham, astro-ph/0105404 to appear in Mon. Not. R. Astron. Soc. (2001),

[2] G. V. Shishkin, Class. Quantum Grav. 8 (1991) 175.

[3] I. I. Cotăescu Mod. Phys. Lett. A 13 (1998) 2991.

[4] S. Weinberg, The Quantum Theory of Fields (Univ. Press, Cambridge, 1995)

[5] I. I. Cotăescu J. Phys. A: Math. Gen. 33 (2000) 9177.

[6] B. Carter and R. G. McLenaghan Phys. Rev. D 19 (1979) 1093

[7] S. Weinberg, Gravitation and Cosmology: Principles and Applications of the General Theory of Relativity (Wiley, New York, 1972)

[8] R. M. Wald, General Relativity, (Univ. of Chicago Press, Chicago and London, 1984)

[9] W.-K. Tung, Group Theory in Physics (World Sci., Philadelphia, 1985)

[10] B. Thaller, The Dirac Equation (Springer Verlag, Berlin Heidelberg, 1992)

[11] N. D. Birrel and P. C. W. Davies, Quantum Fields in Curved Space (Univ. Press, Cambridge, 1982)

[12] M. Abramowitz and I. A. Stegun, Handbook of Mathematical Functions (Dover, 1964) 